\title{
The Simplified Quasi-Optimal Estimates of the Time and Power Parameters of a Low-Frequency Random Pulse with Arbitrary Modulating Function
}

\author{
Oleg V. Chernoyarov ${ }^{1,2, *}$, Alexandra V. Salnikova ${ }^{3}$, Alexander N. Faulgaber ${ }^{1}$ and Boris I. Shakhtarin ${ }^{4}$ \\ ${ }^{1}$ Russia, 111250, Moscow, Krasnokazarmennaya st., 14, National Research University "Moscow Power Engineering Institute" \\ ${ }^{2}$ Russia, 634050, Tomsk, Lenin avenue, 36, International Laboratory of Statistics of Stochastic Processes and Quantitative \\ Finance of National Research Tomsk State University \\ ${ }^{3}$ Russia, Voronezh, 20th Anniversary of October st., 84, Voronezh State University of Architecture and Civil Engineering \\ ${ }^{4}$ Russia, 105005, Moscow, Baumanskaya 2-ya st., 5, Bauman Moscow State University \\ ${ }^{*}$ Corresponding author
}

\begin{abstract}
We introduce a simpler approach for obtaining of usable processing algorithms of fast fluctuating Gaussian pulses with arbitrary modulation function in conditions of parametric prior uncertainty. We carry out the synthesis and analysis of the quasi-optimal measurer of a low-frequency random pulse signal with unknown appearance time, mathematical expectation and dispersion. We find the asymptotically exact expressions for the conditional biases and variances of the resulting estimates. By methods of statistical computer modeling the usefulness and efficiency of the considered technique is corroborated, the working capacity of offered measurer is established, and applicability borders of asymptotically exact formulas for its characteristics are also defined.
\end{abstract}

Keywords-fast fluctuating random signal; maximum likelihood method; decision statistics; unknown parameters; Cramer-Rao bound; quasi-optimal estimation; local Markov approximation method; statistical modeling

\section{INTRODUCTION}

We are going to use a low-frequency random pulse signal with arbitrary modulating function to mean the multiplicative combination of the form of [1-3, et al.]

$$
s(t)=\xi(t) f\left[\gamma\left(t-\lambda_{0}\right)\right] I\left(\frac{t-\lambda_{0}}{\tau}\right), I(x)=\left\{\begin{array}{l}
1,|x| \leq 1 / 2, \\
0,|x|>1 / 2 .
\end{array}\right.
$$

here $\lambda_{0}$ is the appearance time, $\tau$ is the duration of a signal, $f(t)$ is the modulating function (envelope), which is describing the pulse form and is normalized so that $\max f(t)=1, \gamma$ is a scale coefficient, and $\xi(t)$ is the realization of the stationary centered Gaussian random process with mathematical expectation (ME) $\langle\xi(t)\rangle=a_{0}$ and spectral density

$$
G(\omega)=\left(2 \pi D_{0} / \Omega\right) I(\omega / \Omega)
$$

In Eq. (2) the designations are the following: $\Omega$ is the bandwidth, and $D_{0}$ is the dispersion of the process $\xi(t)$.

We presuppose that fluctuations $\xi(t)$ are "fast", that is the pulse duration $\tau$ and the characteristic changing time $\Delta t$ of the function $f(t)$ essentially exceed the correlation time of the process $\xi(t)$, so the following conditions are satisfied

$$
\tau>>4 \pi / \Omega \quad(\mu=\tau \Omega / 4 \pi>>1), \quad \Delta t>>4 \pi / \Omega
$$

In [1] the estimation problem of the appearance time $\lambda_{0} \in\left[\Lambda_{1}, \Lambda_{2}\right]$ of a signal (1) observed against Gaussian white noise with one-sided spectral density $N_{0}$ is considered provided that all other pulse parameters are a priori known. However, in a number of practical tasks ME $a_{0}$ and dispersion $D_{0}$ of the process $\xi(t)$ can also be unknown. In this connection, it is of interest to find the structure and characteristics of the feasible measurer of time and power parameters of a signal (1).

\section{The Estimation ALGORITHM SYNTHESIS}

When synthesizing the estimation algorithm we use a maximum likelihood method [3-5]. Under this method, it is necessary to form the decision statistics represented by the logarithm of the functional of likelihood ratio (FLR) as the function $L(\lambda, a, D)$ of current values $\lambda, a, D$ of the unknown parameters $\lambda_{0}, a_{0}, D_{0}$. If the conditions (3) are fulfilled, then according to $[1,2]$ we have

$$
\begin{gathered}
L(\lambda, a, D)=\frac{q}{N_{0}} \int_{\lambda-\tau / 2}^{\lambda+\tau / 2} \frac{f^{2}[\gamma(t-\lambda)] y^{2}(t)}{1+q f^{2}[\gamma(t-\lambda)]} d t+ \\
+\frac{2 a}{N_{0}} \int_{\lambda-\tau / 2}^{\lambda+\tau / 2} \frac{f[\gamma(t-\lambda)] x(t)}{1+q f^{2}[\gamma(t-\lambda)]} d t- \\
-\frac{a^{2}}{N_{0}} \int_{-\tau / 2}^{\tau / 2} \frac{f^{2}(\gamma t)}{1+q f^{2}(\gamma t)} d t+\frac{\Omega}{4 \pi} \int_{-\tau / 2}^{\tau / 2} \ln \left[1+q f^{2}(\gamma t)\right] d t,
\end{gathered}
$$


where $q=D / E_{N}, E_{N}=N_{0} \Omega / 4 \pi$ is the average power of noise $n(t)$ within bandwidth of the process $\xi(t)$, and $y(t)=\int_{-\infty}^{\infty} x\left(t^{\prime}\right) h\left(t-t^{\prime}\right) d t^{\prime}$ is output signal (response) of the filter with transfer function $H(\omega)$, which satisfies to a condition $|H(\omega)|^{2}=I(\omega / \Omega)$, on the observable data realization $x(t)=s(t)+n(t)$.

Then maximum likelihood estimates (MLEs) $\lambda_{m}, a_{m}$ and $D_{m}$ of the appearance time $\lambda_{0}$, ME $a_{0}$ and dispersion $D_{0}$ of a random pulse (1) are determined as the position of the greatest maximum of decision statistics $L(\lambda, a, D)$ :

$$
\begin{gathered}
\lambda_{m}=\underset{\lambda \in\left[\Lambda_{1}, \Lambda_{2}\right]}{\operatorname{argsup}} L\left(\lambda, a_{m}, D_{m}\right), \\
\left(a_{m}, D_{m}\right)=\underset{a \in(-\infty, \infty), D \geq 0}{\arg \sup } L\left(\lambda_{m}, a, D\right) .
\end{gathered}
$$

It is easy to see that the measurer (5) has multichannel structure and the infinitely many channels are required for its exact implementation that it is hardly probably in practice. Thereupon, it might be useful to find single-channel quasioptimal estimation algorithms of time and power parameters of a signal (1) close to the optimal algorithm (5) by their accuracy characteristics.

Similarly to [5] it can be shown that MLE $\lambda_{m} \rightarrow \lambda_{0}$ in mean square, if $\mu \rightarrow \infty$. Then, according to [6], the characteristics of MLEs $a_{m}$ and $D_{m}$ (5) coincide asymptotically (with increasing $\mu$ ) with characteristics of the estimates

$$
\left(a_{m 0}, D_{m 0}\right)=\underset{a \in(-\infty, \infty), D \geq 0}{\arg \sup } L\left(\lambda_{0}, a, D\right)
$$

In general, the measurer (6) has also multichannel organization, while the minimum variances $V_{a_{\min }}$ and $V_{D_{\min }}$ of the estimates $a_{m 0}$ and $D_{m 0}(6)$ determined by the Cramer-Rao formula [4] are equal to

$$
\begin{aligned}
& V_{a_{\min }}=-1 /\left.\left\langle d^{2} L\left(\lambda_{0}, a, D_{0}\right) / d a^{2}\right\rangle\right|_{a=a_{0}}=E_{N} / 2 \mu G_{21}, \\
& V_{D_{\min }}=-1 /\left.\left\langle d^{2} L\left(\lambda_{0}, a_{0}, D\right) / d D^{2}\right\rangle\right|_{D=D_{0}}= \\
& \quad=E_{N}^{2} /\left[\mu G_{42}+\left(G_{42}^{2}-2 G_{21} G_{63}\right) / G_{21}^{2}\right] .
\end{aligned}
$$

here $G_{m n}=\int_{-1 / 2}^{1 / 2} f^{m}(\tilde{\gamma} \tilde{t}) d \tilde{t} /\left[1+q_{0} f^{2}(\tilde{\gamma} \tilde{t})\right]^{n}, q_{0}=D_{0} / E_{N}$, $\tilde{\gamma}=\tau \gamma$ and $\tilde{t}=t / \tau$ is the normalized time. As it is noted in [4], the variances of MLEs (6) coincide asymptotically (with increasing an output signal-to-noise ratio (SNR)) with Eqs. (7).
We introduce some simpler quasi-optimal estimates (QOEs) $a_{q 0}$ and $D_{q 0}$ of the pulse (1) ME $a_{0}$ and dispersion $D_{0}$ instead of MLEs $a_{m 0}$ and $D_{m 0}(6)$. In the synthesis of QOEs $a_{q 0}$ and $D_{q 0}$ we use the condition for closeness of their variances to the minimum variances (7) provided that QOEs can be technically implemented by single-channel units. Besides, in some limiting cases QOEs $a_{q 0}$ and $D_{q 0}$ should move to MLEs $a_{m 0}$ and $D_{m 0}(6)$. As a result, we come to the estimates in terms of

$$
\begin{gathered}
a_{q 0}=L_{2}\left(\lambda_{0}\right) / \tau G_{10}, \\
D_{q 0}=\max \left[0,\left(L_{1}\left(\lambda_{0}\right) / \tau-E_{N}\right) / G_{20}-\left(L_{2}\left(\lambda_{0}\right) / \tau G_{10}\right)^{2}\right],
\end{gathered}
$$

where $L_{1}(\lambda)=\int_{\lambda-\tau / 2}^{\lambda+\tau / 2} y^{2}(t) d t, L_{2}(\lambda)=\int_{\lambda-\tau / 2}^{\lambda+\tau / 2} x(t) d t$, and $E_{N}$, $y(t)$ are determined the same as in Eq. (4).

Carrying out in Eqs. (8) the averaging through all possible realizations $x(t)$ (with fixed values of all unknown parameters), for conditional biases $b\left(a_{q 0} \mid a_{0}\right), b\left(D_{q 0} \mid D_{0}\right)$ and variances $V\left(a_{q 0} \mid a_{0}\right), V\left(D_{q 0} \mid D_{0}\right)$ of QOEs $a_{q 0}, D_{q 0}$ we get

$$
\begin{gathered}
b\left(a_{q 0} \mid a_{0}\right)=0, \quad V\left(a_{q 0} \mid a_{0}\right)=E_{N}\left(1+q_{0} G_{20}\right) / 2 \mu G_{10}^{2}, \\
b\left(D_{q 0} \mid D_{0}\right)=-E_{N}\left(1+q_{0} G_{20}\right) / 2 \mu G_{10}^{2}, \\
V\left(D_{q 0} \mid D_{0}\right)=\frac{E_{N}^{2}}{\mu}\left\{\frac{1}{G_{10}^{2}} \int_{-1 / 2}^{1 / 2}\left[1+q_{0} f^{2}(\tilde{\gamma} \tilde{t})\right]^{2} d \tilde{t}-\right. \\
-\frac{1}{\mu G_{20}}\left[\frac{1}{G_{10}} \int_{-1 / 2}^{1 / 2}\left[1+q_{0} f^{2}(\tilde{\gamma} \tilde{t})\right]^{2} d \tilde{t}-\frac{3\left(1+q_{0} G_{20}\right)^{2}}{4 G_{20}}\right]+ \\
\left.+\eta^{2}\left[\frac{1}{G_{20}}-\frac{1}{G_{10}}+q_{0} \int_{-1 / 2}^{1 / 2} f^{2}(\tilde{\gamma} \tilde{t})\left(\frac{1}{\sqrt{G_{20}}}-\frac{f(\tilde{\gamma} \tilde{t})}{G_{10}}\right)^{2} d \tilde{t}\right]\right\} .
\end{gathered}
$$

here $\eta^{2}=2 a_{0}^{2} / E_{N}$ and $q_{0}$ is determined the same as in Eq. (7).

As follows from Eqs. (7), (9), with fulfillment of conditions (3) the variances of the estimates $a_{q 0}, D_{q 0}$ (8) differs from ultimate variances by no more than $5 \%$ for a large class of modulating functions $f(t)$. If $f(t) \equiv 1$, then variances (7) and (9) coincide, i.e. QOEs (8) converges to MLEs (6) with approach of the form of the modulating function $f(t)$ to rectangular. It allows us to recommend the single-channel algorithm (8) instead of more complex multichannel (6) to measure the pulse signal (1) ME and dispersion in practical applications without significant loss in accuracy of a passed estimate. 
If the parameter $\lambda_{0}$ is unknown, then from Eq. (8) we obtain the estimates of the kind of

$$
\begin{gathered}
a_{q}=L_{2}\left(\lambda_{q}\right) / \tau G_{10}, \\
D_{q}=\max \left[0,\left(L_{1}\left(\lambda_{q}\right) / \tau-E_{N}\right) / G_{20}-\left(L_{2}\left(\lambda_{q}\right) / \tau G_{10}\right)^{2}\right],
\end{gathered}
$$

where $\lambda_{q}=\underset{\lambda \in\left[\Lambda_{1}, \Lambda_{2}\right]}{\operatorname{argsup}} L\left(\lambda, a_{q}, D_{q}\right)$ is the estimate of the appearance time of a pulse (1). Substituting Eq. (10) in Eq. (4) and, following [7], carrying out optimization of the estimation algorithm we move to the estimate of the appearance time

$$
\begin{gathered}
\lambda_{q}=\underset{\lambda \in\left[\Lambda_{1}, \Lambda_{2}\right]}{\operatorname{argsup}} L_{q}(\lambda), \\
L_{q}(\lambda)=\mu\left\{L_{1}(\lambda) / \tau E_{N}-\ln \left[\left(L_{1}(\lambda)-f^{2}(\gamma \tau / 2) L_{2}^{2}(\lambda) / \tau\right) / \tau E_{N}\right]-1\right\}
\end{gathered}
$$

instead of the estimate $\lambda_{m}$ (5). We also name the estimates (10), (11) as QOEs. Indeed, if $f(t) \equiv 1$, then QOEs (10), (11) go over the corresponding MLEs of the ME, dispersion and appearance time of a low-frequency random pulse with rectangular modulating function [8].

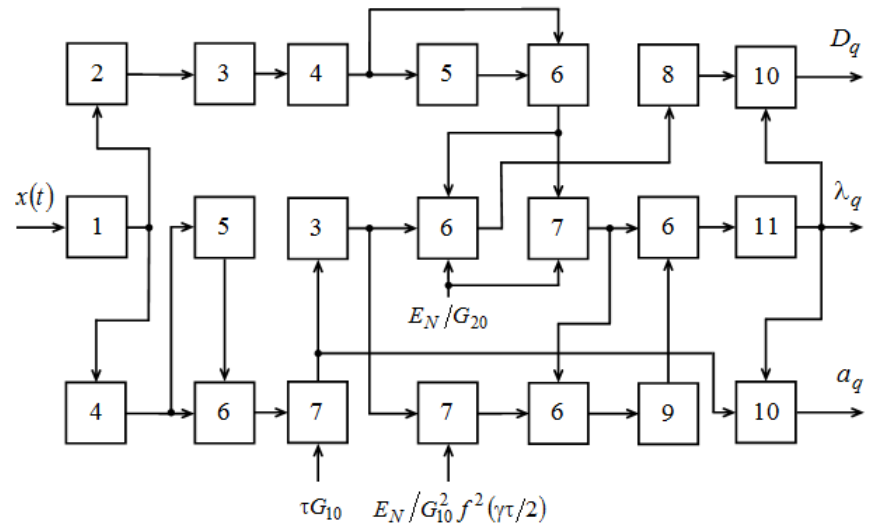

FIGURE I. THE QUASI-OPTIMAL MEASURER OF THE APPEARANCE TIME, MATHEMATICAL EXPECTATION AND DISPERSION OF A LOW-FREQUENCY RANDOM PULSE WITH ARBITRARY MODULATING FUNCTION

The measurer (10), (11) of the time and power parameters of a pulse signal (1) can be implemented on the basis of the block diagram presented in Figure 1. Here are the designations: 1 is the switch that is open for time $\left[\Lambda_{1}-\tau / 2, \Lambda_{2}+\tau / 2\right], 2$ is a filter with transfer function $H(\omega) / \sqrt{\tau G_{20}}$ (4), 3 is the squarer, 4 is an integrator, 5 is a delay line for the period $\tau, 6$ is the substracter, 7 is the divider, 8 is the nonlinear element with characteristic $\max (0, x), 9$ is the logarithmic amplifier, 10 is the sampling device forming to its output the input signal sample at the instant time $t=\lambda_{q}+\tau / 2,11$ is the retriever of the location of the input signal greatest maximum (extremator).

\section{The EstimATION ALgORITHM ANALYSIS}

Let us find the characteristics of the estimates (10), (11). During the analysis process, we find it expedient to divide all QOEs (11) of the pulse appearance time into the two classes: reliable and anomalous $[4,5]$. The estimate $\lambda_{q}$ is reliable, if it is within the interval limits $\Gamma_{S} \equiv\left[\lambda_{0}-\tau, \lambda_{0}+\tau\right]$. If QOE $\lambda_{q}$ is out of an interval $\Gamma_{S}$, i.e. $\lambda_{q} \in \Gamma_{N}=\left[\Lambda_{1}, \lambda_{0}-\tau\right) \cup\left(\lambda_{0}+\tau, \Lambda_{2}\right]$, then the estimate and the corresponding estimate error are designated as anomalous $[1,2,5]$. It is necessary to consider the anomalous errors, if the length of the prior interval of the possible values of appearance time $\lambda_{0}$ is much greater than the length of the interval $\Gamma_{S}$ of the reliable estimate, i.e. the following condition holds

$$
m=\left(\Lambda_{2}-\Lambda_{1}\right) / \tau>>1
$$

According to $[4,5]$, while executing ratio (16), the conditional bias $b\left(\lambda_{q} \mid \lambda_{0}\right)=\left\langle\lambda_{q}-\lambda_{0}\right\rangle$ and variance $V\left(\lambda_{q} \mid \lambda_{0}\right)=\left\langle\left(\lambda_{q}-\lambda_{0}\right)^{2}\right\rangle$ of the estimate $\lambda_{q}$, with the allowance for the anomalous errors, can be written down as follows:

$$
\begin{gathered}
b\left(\lambda_{q} \mid \lambda_{0}\right)=P_{0} b_{0}\left(\lambda_{q} \mid \lambda_{0}\right)+\left(1-P_{0}\right)\left[\left(\Lambda_{1}+\Lambda_{2}\right) / 2-\lambda_{0}\right], \\
V\left(\lambda_{q} \mid \lambda_{0}\right)=P_{0} V_{0}\left(\lambda_{q} \mid \lambda_{0}\right)+ \\
+\left(1-P_{0}\right)\left[\left(\Lambda_{1}^{2}+\Lambda_{1} \Lambda_{2}+\Lambda_{2}^{2}\right) / 3-\lambda_{0}\left(\Lambda_{1}+\Lambda_{2}\right)+\lambda_{0}^{2}\right] .
\end{gathered}
$$

here $b_{0}\left(\lambda_{q} \mid \lambda_{0}\right), \quad V_{0}\left(\lambda_{q} \mid \lambda_{0}\right), \quad P_{0}=P\left[\left|\lambda_{q}-\lambda_{0}\right| \leq \tau\right]$ are, correspondingly, conditional bias, conditional variance and probability of a reliable estimate $\lambda_{q}$ (11).

While determining $b_{0}\left(\lambda_{q} \mid \lambda_{0}\right), V_{0}\left(\lambda_{q} \mid \lambda_{0}\right)$ and $P_{0}$, we will be limited to a condition of a high posterior accuracy, when the output power SNR of the algorithm (10), (11) is sufficiently great. We also consider that $f(\gamma t)$ is even function (for simplicity of overall expressions) and does not vanish in points $t= \pm \gamma \tau / 2$ (the useful signal (1) is discontinuous [5]). Then, based on the results of works [2, 8, 9] we obtain

$$
\begin{aligned}
& P_{0} \approx \frac{2 \psi z}{\sigma \sqrt{\mu}} \exp \left(\frac{\psi^{2} z^{2}}{2}+\psi z^{2}\right) \int_{H}^{\infty} \exp \left[-m \phi\left(\sqrt{\frac{u}{2}}, \frac{1}{f(\tilde{\gamma} / 2)}\right)\right] \times \\
& \quad \times\left\{\exp \left(-\frac{\psi z u}{\sigma \sqrt{\mu}}\right) \Phi\left[\frac{u}{\sigma \sqrt{\mu}}-z(\psi+1)\right]-\right. \\
& \left.-\exp \left[\frac{3 \psi^{2} z^{2}}{2}+\psi z\left(z-\frac{2 u}{\sigma \sqrt{\mu}}\right)\right] \Phi\left[\frac{u}{\sigma \sqrt{\mu}}-z(2 \psi+1)\right]\right\} d u
\end{aligned}
$$




$$
\begin{gathered}
b_{0}\left(\lambda_{q} \mid \lambda_{0}\right)=0, \\
V_{0}\left(\lambda_{q} \mid \lambda_{0}\right)=\frac{13 \tau^{2}}{8} \frac{\left\{\left[1+q_{0} f^{2}(\tilde{\gamma} / 2)\left(1+\left(q_{0}+\eta^{2}\right) f^{2}(\tilde{\gamma} / 2)\right)\right] \times\right.}{\mu^{2} f^{4}(\tilde{\gamma} / 2) \times} \rightarrow \\
\rightarrow \frac{\times\left[q_{0} G_{20}+\left(\eta^{2} / 2\right)\left(G_{20}-G_{10}^{2} f^{2}(\tilde{\gamma} / 2)\right)\right]^{2}+}{\times\left\{\eta^{2} G_{10} f(\tilde{\gamma} / 2)+\left(q_{0}+\eta^{2} / 2\right) \times\right.} \rightarrow \\
\rightarrow \frac{+\eta^{2} G_{10} f^{3}(\tilde{\gamma} / 2)\left[G_{10} f(\tilde{\gamma} / 2)+\left(1+q_{0} f^{2}(\tilde{\gamma} / 2)\right) \times\right.}{\times\left[q_{0} G_{20}+\left(\eta^{2} / 2\right) \times\right.} \rightarrow \\
\rightarrow \frac{\left.\times\left(G_{10} f(\tilde{\gamma} / 2)+2 q_{0} G_{20}+\eta^{2}\left(G_{20}-G_{10}^{2} f^{2}(\tilde{\gamma} / 2)\right)\right]\right\}^{2}}{\left.\left.\times\left(G_{20}-G_{10}^{2} f^{2}(\tilde{\gamma} / 2)\right)\right]\right\}^{4}} .
\end{gathered}
$$

here

$$
\begin{aligned}
& H=\frac{2 f(\tilde{\gamma} / 2)}{\sqrt{1+f^{2}(\tilde{\gamma} / 2)}}, z^{2}=\frac{\mu}{\sigma^{2}}\left[G_{20}\left(q_{0}+\eta^{2} / 2\right)-\ln (1+Q)\right]^{2}, \\
& \psi=\frac{2 \sigma A_{S} \sqrt{\mu}}{z\left(\sigma_{1}^{2}+\sigma_{2}^{2}\right)}, A_{S}=\frac{f^{2}(\tilde{\gamma} / 2)}{1+Q}\left[Q\left(q_{0}+\eta^{2} / 2\right)+\eta^{2} G_{10} f(\tilde{\gamma} / 2)\right] \text {, } \\
& \sigma^{2}=\left\{Q^{2}\left[1+q_{0} G_{20}+\left(G_{20}+q_{0} G_{40}\right)\left(q_{0}+\eta^{2}\right)\right]+2 \eta^{2} Q G_{10} \times\right. \\
& \left.\times f^{2}(\tilde{\gamma} / 2)\left(G_{10}+q_{0} G_{30}\right)+\eta^{2} G_{10}^{2} f^{4}(\tilde{\gamma} / 2)\left(1+q_{0} G_{20}\right)\right\} /(1+Q)^{2} \text {, } \\
& \sigma_{1}^{2}=\left[1+q_{0} f^{2}(\tilde{\gamma} / 2)\right]\left\{Q^{2}\left[1+\left(q_{0}+\eta^{2}\right) f^{2}(\tilde{\gamma} / 2)\right]+\right. \\
& \left.+\eta^{2} f^{3}(\tilde{\gamma} / 2) G_{10}\left[G_{10} f(\tilde{\gamma} / 2)+2 Q\right]\right\} /(1+Q)^{2}, \\
& \sigma_{2}^{2}=\frac{Q^{2}+\eta^{2} G_{10}^{2} f^{4}(\tilde{\gamma} / 2)}{(1+Q)^{2}}, Q=q_{0} G_{20}+\frac{\eta^{2}}{2}\left[G_{20}-G_{10}^{2} f^{2}(\tilde{\gamma} / 2)\right] \text {, } \\
& \phi(h, \gamma)=\left\{4 h^{2}\left[1+\gamma^{2}-\left(\gamma^{2}-1\right) I_{1}\left(h^{2}\left(\gamma^{2}-1\right)\right) / I_{0}\left(h^{2}\left(\gamma^{2}-1\right)\right)\right]-1\right\} \times \\
& \times \gamma I_{0}\left[h^{2}\left(\gamma^{2}-1\right)\right] \exp \left[-h^{2}\left(\gamma^{2}+1\right)\right],
\end{aligned}
$$

Now we consider the characteristics of QOEs $a_{q}$ and $D_{q}$ (10). It follows from formulas (9) that when $\lambda_{q}=\lambda_{0}$ and the conditions (3) are fulfilled, the variances of the estimates (10) are not decreasing faster than $\mu^{-1}$. At the same time, the variance (15) of the estimate $\lambda_{q}$ (11) is proportional $\mu^{-2}$. Therefore, similarly to [6] it can be shown that with $\mu>>1$, $z>>1(\operatorname{SNR}>>1)$, when $P_{0} \approx 1$ and the estimate $\lambda_{q}$ is reliable, the characteristics of the estimates $a_{q}$ and $D_{q}(10)$ coincide with the characteristics (9) found with the known value of the parameter $\lambda_{0}$. The accuracy of formulas (9) increases with $\mu$ and $z$.

Assuming in Eqs. (9) $f(t) \equiv 1$, we obtain the expressions for conditional biases and variances of MLEs of the ME and dispersion of a rectangular random pulse (1) with unknown appearance time [8].

\section{The Results of Statistical Modeling}

For the purpose of an experimental functional test of the introduced quasi-optimal simultaneous estimation algorithm of the appearance time, ME and dispersion of a random pulse (1) and an establishment of the applicability borders of the asymptotically exact formulas (9), (13)-(15) for its characteristics, we applied the statistical computer modeling of the measurer (10), (11). The modeling technique of processing algorithms of random pulse signals is described in detail in [9].

Some results of statistical modeling for $\lambda_{0}=\left(\Lambda_{2}+\Lambda_{1}\right) / 2$, $\Lambda_{1}=\tau / 2, \Lambda_{2}=(m+1 / 2) \tau, f(t)=\exp \left(-\gamma^{2} t^{2}\right), \gamma=1 / \tau$ and $z_{0}^{2}=2 a_{0}^{2} \tau / N_{0}=10$ are shown in Figures 2-4. Each experimental value in Figures 2-4 was obtained as a result of the processing of no less than $10^{4}$ realizations of the observable data $x(t)$. So, with probability of 0.9 confidence intervals boundaries deviate from experimental values no more than for $10 \ldots 15 \%$.

In Figure 2 solid lines represent dependences (13) of the normalized conditional variance $\widetilde{V}_{\lambda}\left(q_{0}\right)=12 V\left(\lambda_{q} \mid \lambda_{0}\right) /\left(\Lambda_{2}-\Lambda_{1}\right)^{2}$ of QOE $\lambda_{q}$ (11) from parameter $q_{0}$ (7) taking into account anomalous errors, if $m=20$ (12). Here analogous dependences (15) of the normalized variance $\widetilde{V}_{0 \lambda}\left(q_{0}\right)=12 V_{0}\left(\lambda_{q} \mid \lambda_{0}\right) /\left(\Lambda_{2}-\Lambda_{1}\right)^{2}$ of the reliable QOE $\lambda_{q}$ (11) are also drawn by dashed lines. Curves 1 are calculated with $\mu=50,2-100,3-200$. The experimental values for $\mu=50,100$ and 200 are denoted by rectangles, crosses, and rhombuses (for variance $\widetilde{V}_{\lambda}$ of the estimate $\lambda_{q}$ with the allowance for anomalous errors), respectively, as well as by circles, triangles and asterisks (for variance $\widetilde{V}_{0 \lambda}$ of the reliable estimate $\lambda_{q}$ ). 


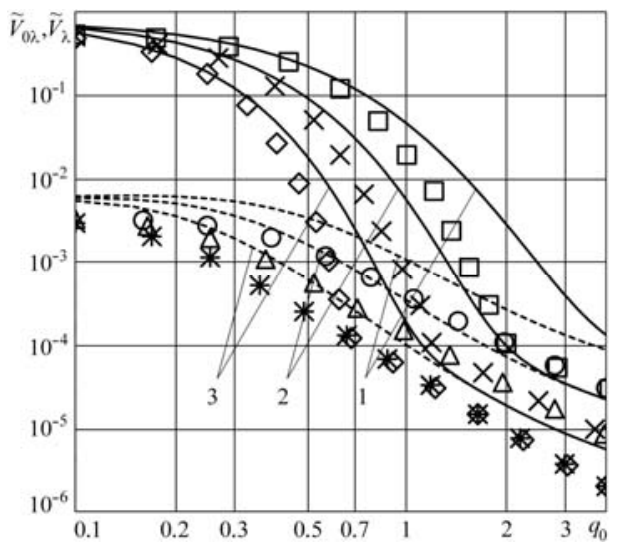

FIGURE II. THE NORMALIZED VARIANCE OF THE ESTIMATE OF THE PULSE APPEARANCE TIME

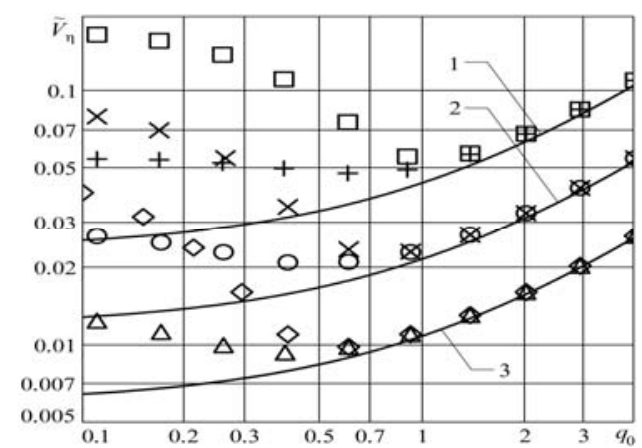

FIGURE III. THE NORMALIZED VARIANCE OF THE ESTIMATE OF THE PULSE MATHEMATICAL EXPECTATION.

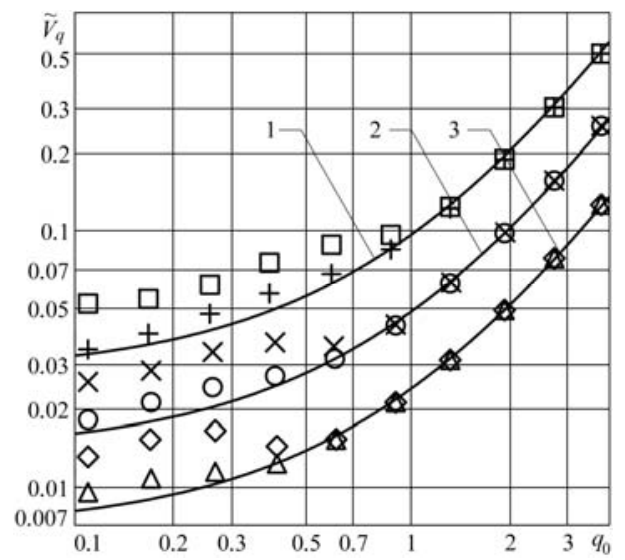

FIGURE IV. THE NORMALIZED VARIANCE OF THE ESTIMATE OF THE PULSE DISPERSION

In Figures 3, 4 the theoretical dependences (9) of the normalized conditional variances $\tilde{V}_{\eta}\left(q_{0}\right)=2 V\left(a_{q} \mid a_{0}\right) / E_{N}$, $\tilde{V}_{q}=V\left(D_{q} \mid D_{0}\right) / E_{N}^{2}$ of the estimates $a_{q}, D_{q}(10)$ are traced by solid lines. Curves 1 correspond to $\mu=50,2-100,3-200$. Experimental values of variances $\widetilde{V}_{\eta} \widetilde{V}_{q}$ in Figures 3, 4 are shown by squares, crosses, rhombuses, when $m=2$ and the estimate of the appearance time $l_{q}$ is reliable, and by pluses, circles, triangles, when $m=20$ and anomalous errors are possible measuring the appearance time.

As is evident from Figures 2-4, the theoretical dependences (9), (13)-(15) for characteristics of the measurer (10), (11) of time and power parameters of a random pulse (1) approximate the experimental results in a satisfactory manner, at least, for $m \geq 20, \mu \geq 50, z \geq 1.5 \ldots 2,\left(f_{\max }-f_{\min }\right) / \mu<4 \cdot 10^{-3}$. Here $f_{\min }=\min f(t)$ and $f_{\max }=1$ are minimum and maximum values of the function $f(t)$.

So, the obtained results make it possible to perform a valid choice between offered and other estimation algorithms of the appearance time, ME and dispersion of a low-frequency random pulse signal with arbitrary modulation function depending on the available prior information and the required accuracy and simplicity of hardware measurer implementation.

\section{CONCLUSION}

Optimal measurers of several unknown parameters of random pulse signals designed with help of the traditional (maximum likelihood, Bayesian) approaches have sufficiently complex multichannel structure. In order to obtain the effective single-channel estimation algorithms the technique based on closeness of accuracy of formed estimates of continuous signal parameters to the potential accuracy (Cramer-Rao bound) can be used. Application of the specified technique allows us to synthesize the quasi-optimal estimation algorithm of the appearance time of a Gaussian pulse which is invariant to the mathematical expectation and dispersion of a useful signal, as well as the form of its modulating function. At the same time, the characteristics of quality rating of the introduced algorithm are comparable to the corresponding characteristics of the technically more complex maximum likelihood estimation algorithm.

To implement the quasi-optimal measurer of the power parameters of a random pulse with unknown appearance time we need to know only the first and second moments of modulating function. Accuracy of the quasi-optimal estimates of mathematical expectation and dispersion has almost no differs from accuracy of the corresponding maximum likelihood estimates for a wide class of modulating functions. Conclusions and recommendations are valid, if the output signal-to-noise ratio is greater than $2 \ldots 3$.

\section{ACKNOWEDGEMENT}

The reported study was supported by the Ministry of Education and Science of the Russian Federation (research project No. 1729) and Russian Foundation for Basic Research (research projects No. 16-01-00121)

\section{REFERENCES}

[1] O.V. Chernoyarov, M.F. Rashitov, "The efficiency of reception of a random untailored pulse with unknown appearance time" [in Russian], Herald of the Moscow Power Engineering Institute, No. 5, 2010, pp. 102-110.

[2] O. V. Chernoyarov, M. Vaculik M., A. Shirikyan, A.V. Salnikova, "Statistical analysis of fast fluctuating random signals with arbitrary- 
function envelope and unknown parameters", Komunikacie, vol. 17, No.

1A, 2015, pp. 35-43.

[3] H.L. van Trees, K.L. Bell, Z. Tian, Detection, Estimation, and Modulation Theory. Part I. Detection, Estimation and Filtering Theory. Wiley, New York, 2013.

[4] E.I. Kulikov, A.P. Trifonov, Estimation of Signal Parameters against Hindrances [in Russian]. Sovetskoe Radio, Moscow, 1978.

[5] A.P. Trifonov, Yu.S. Shinakov, Joint Discrimination of Signals and Estimation of Their Parameters against Background [in Russian]. Radio i Svyaz', Moscow, 1986.

[6] Basseville M., Benveniste A. et al., Detection of Abrupt Changes in Signals and Dynamical Systems. Lecture Notes in Control and Information Sciences, LNCIS-77. Springer-Verlag, Berlin, 1985.

[7] A.V. Zakharov, "Optimization of detection algorithm of fluctuated radiopulse with unknown time delay" [in Russian], Proceedings of Voronezh State University. Series: Physics. Mathematics, No. 1, 2005, pp. 46-56.

[8] A.P. Trifonov, A.V. Zakharov, V.I. Parfenov, "Efficiency of reception of a random pulse signal with unknown parameters", Radiotekhnika i Elektronika, vol. 36, No. 7, 1991, pp. 1300-1308.

[9] O.V. Chernoyarov, Sai Si Thu Min, A.V. Salnikova, B.I. Shakhtarin, A.A. Artemenko, "Application of the Local Markov Approximation Method for the Analysis of Information Processes Processing Algorithms with Unknown Discontinuous Parameters", Applied Mathematical Sciences, vol. 8, No. 90, 2014, pp. 4469-4496. 